\title{
Mathematical description of the nonlinear chemical reactions with oscillatory inflow to the reaction field
}

\author{
ALDONA KRUPSKA \\ Institute of Molecular Physics PAS, M. Smoluchowskiego 17, 60-179 Poznań, Poland \\ e-mail: aldona.krupska@ifmpan.poznan.pl
}

MS received 5 January 2015; revised 3 February 2015; accepted 3 February 2015

\begin{abstract}
In this paper the arduous attempt to find a mathematical solution for the nonlinear autocatalytic chemical processes with a time-varying and oscillating inflow of reactant to the reaction medium has been taken. Approximate analytical solution is proposed. Numerical solutions and analytical attempts to solve the non-linear differential equation indicates a phase shift between the oscillatory influx of intermediate reaction reagent $X$ to the medium of chemical reaction and the change of its concentration in this medium. Analytical solutions indicate that this shift may be associated with the reaction rate constants $k_{1}$ and $k_{2}$ and the relaxation time $\tau$. The relationship between the phase shift and the oscillatory flow of reactant $X$ seems to be similar to that obtained in the case of linear chemical reactions, as described previously, however, the former is much more complex and different. In this paper, we would like to consider whether the effect of forced phase shift in the case of nonlinear and non-oscillatory chemical processes occurring particularly in the living systems have a practical application in laboratory.
\end{abstract}

Keywords. Autocatalytic reactions; oscillatory inflow; forced phase delay effect; approximate solution.

PACS Nos $*$ 43.25.-x, 43.25. $+\mathrm{y} ;$ 05.45.-a; 02.30.Ks

\section{Introduction}

Nonlinear chemical reactions, especially autocatalytic type, have been studied experimentally by many researchers. ${ }^{1,2}$ Reactions of this type can be reversible or irreversible, with or without the inflow of the intermediate product $X$. Many processes in nature are autocatalytic, such as the self-replication exhibited by ligase ribozyme ${ }^{3}$ and peptides, ${ }^{4}$ self-perpetuating conformational changes such as those known to be exhibited by prions ${ }^{5}$ or reproduction events in biological populations. ${ }^{6}$ The effect of a reversible autocatalytic process of spontaneous decay of the catalyst population was considered. ${ }^{7}$ The authors consider the system for which the spontaneous production of the autocatalytic reagent is exceedingly rare over the time scale of the experiment. Micro-fluid experiments and blood coagulation have proved that the rate of mixing controls the rate and can regulate the rate and outcome of both biological and non-biological autocatalytic reaction systems. ${ }^{8}$

The autocatalytic stage is involved in many chemical and biochemical cycles. Well known and often considered by many researches are the following: the Belousov-Zhabotynski reactions ${ }^{9-17}$ oxidation of bromates by nitric acid ${ }^{18}$ or Landolt reactions, ${ }^{19}$ ferrousbromate, ${ }^{20}$ iodide - areniate, ${ }^{21}$ chlorite-iodide, and hydroxylamine-nitric acid, ${ }^{22} \mathrm{Fe}(\mathrm{I} 1)-\mathrm{HNO}^{23}$ reactions. Autocatalytic processing of $\beta$-pro'-tryptase to $\beta$-pro'tryptase is described. ${ }^{24}$ These autocatalytic processes possess bistable steady states observed in a stirred tank reactor (CSTR) experiments. ${ }^{25}$ Minimal selfreplicating systems based on autocatalytic processes were analyzed. ${ }^{26}$ Some autocatalytic reactions, for example, may exhibit propagating reaction fronts. ${ }^{27-33}$

A theoretical work is concerned with a model of the enzymatic system of two coupled autocatalytic reactions based on three variables. ${ }^{34-36} \mathrm{~A}$ threevariable model of deterministic chaos in the BelousovZhabotinsky reaction, has been described too. ${ }^{37}$ The autocatalytic stage appears also in the hipercycle model described by Eigen. ${ }^{38}$ A simple three-variable model for complex oscillations with an autocatalytic stage in an isothermal chemical system was considered. ${ }^{39}$ Theoretical approaches used in the Belousov-Zhabotinsky (BZ) reactions ${ }^{40}$ and arsenite-iodate waves, ${ }^{41}$ iodate oxidation, ${ }^{42}$ aerobic oxidation of NADH by horseradish peroxidase enzyme model ${ }^{43}$ and many others. Timeperiodic reaction-diffusion systems with application to the autocatalysis in chemistry were considered too. ${ }^{44}$ The simulation of a complex kinetic scheme and the results verified considering the thermal decomposition of cumene hydroperoxide can be easily generalized 
to different kinetic models and is devoted to identify autocatalytic processes by means of dynamical DSC experiment. ${ }^{45}$ Autocatalytic set concept introduced in 1860 by Darwin was recently used to describe the chemical reactions in combustion process in circulating fluidized bed boiler. ${ }^{46}$

The model of a simple autocatalytic type reaction with oscillatory, cosine-like inflow of intermediate product $X$ to the reaction medium (reaction field) is presented in this paper. Numerical solutions and analytical attempts to solve the non-linear differential equation indicates a phase shift between the oscillatory influx of intermediate reagent $X$ to the reaction medium and change of its concentration in this medium. Attempts for analytical solutions indicate that this shift may be associated with the reaction rate constants $k_{1}$ and $k_{2}$ and the relaxation time $\tau$. The relationship between the phase shift and the oscillatory flow of reactant $X$ seems to be similar to that obtained in the case of linear chemical reactions, as described previously, ${ }^{47}$ however, is much more complex (table 1).

This raises the question: is it possible to use a forced phase shift effect in the simplest nonlinear chemical processes occurring especially in the biological systems, for example DNA replication or formation of thiodiglycol from thioglycolates or that which occurs in the initial transcript of rRNA and many other autoprocessing ones? In this paper, we would like to give an answer to this question. This work is a supplement to our earlier work. ${ }^{47}$

\section{Theory}

Nonlinear reactions with oscillatory inflow of reactant to the reaction medium are considered.

The main subject is the simplest nonlinear autocatalytic reaction with inflow $I_{\mathrm{X}}$ of intermediate reactant to the reaction field:

$$
A+X \stackrel{k_{1} k_{2}}{\longleftrightarrow} 2 X
$$

Where, $A$ stands for the initial product and $X$ is the intermediate product.

The kinetic equation of reaction (1) takes the form:

$$
\frac{d X}{d t}=k_{1} A X-k_{2} X^{2}+I_{X}
$$

We assume that inflow $I_{\mathrm{X}}$ of the intermediate product $X$ is not constant in time, but it changes periodically (cosine-like), according to the following function:

$$
I_{X}=a \cos ^{2}(\omega t)
$$

Where, $a$ is the oscillation amplitude and $\omega$ is the rate (frequency) of $X$ inflow to the reaction medium.

The equation (2) takes the form of:

$$
\frac{d X}{d t}=k_{1} A X-k_{2} X^{2}+a \cos ^{2}(\omega t)
$$

The equation (4) is nonlinear and non-homogenous. The equation (4) has two variables: concentration of

\begin{tabular}{|c|c|c|}
\hline & $\begin{array}{c}\text { Linear reaction type: } \\
\mathrm{A}+\mathrm{X} \leftrightarrow \mathrm{P} \\
\text { with oscillatory inflow of } X^{47}\end{array}$ & $\begin{array}{c}\text { Nonlinear reaction type: } \\
\mathrm{A}+\mathrm{nX} \leftrightarrow(\mathrm{n}+1) \mathrm{X} \\
\text { with oscillatory inflow of } X \text { [this work] }\end{array}$ \\
\hline $\begin{array}{l}\text { analytical solution } \\
\text { delta } \Delta \\
\text { phase shift }\end{array}$ & $\begin{array}{c}\text { strictly possible } \\
\text { time independent } \\
\quad \tan \phi=2 \omega \tau \\
\text { where } \tau=1 / k_{1} A\end{array}$ & $\begin{array}{c}\text { strictly impossible, only default } \\
\text { time dependent } \\
\tan \phi=2 \omega \tau \\
\text { where } \tau=1 / \sqrt{\Delta}\end{array}$ \\
\hline $\begin{array}{l}\text { possibility of determination } \\
\text { a rate constant and a relaxation time }\end{array}$ & possible & impossible \\
\hline $\begin{array}{l}\text { dependence of the phase } \\
\text { shift from supply-frequency } X\end{array}$ & $\begin{array}{l}\text { increases with increasing } \\
\quad \text { flow-frequency } X\end{array}$ & $\begin{array}{l}\text { decreases with increasing } \\
\text { flow-frequency } X\end{array}$ \\
\hline $\begin{array}{l}\text { dependence on initial condition } \\
\text { number of possible behaviours }\end{array}$ & $\begin{array}{l}\text { independent } \\
\text { one }\end{array}$ & $\begin{array}{l}\text { dependent } \\
\text { more than one }\end{array}$ \\
\hline stabilized by the forced delay effect. & stabilized & not stabilized \\
\hline bifurcation point & not occurs & occurs \\
\hline $\begin{array}{l}\text { dependence of the amplitude } \\
\text { on frequency of } X \text { inflow }\end{array}$ & $\begin{array}{c}\text { decreases with increases of } X \\
\text { inflow frequency }\end{array}$ & $\begin{array}{l}\text { decreases with increases of } X \\
\text { inflow frequency or does not change }\end{array}$ \\
\hline $\begin{array}{l}\text { influence of increasing frequency } \\
\text { of } X \text { inflow on the phase shift }\end{array}$ & $\begin{array}{l}\text { tends to } \pi / 2 \text { but cannot } \\
\text { exceed this value }\end{array}$ & tends to zero \\
\hline
\end{tabular}
intermediate product $X$ and time variable $t$. For this

Table 1. Comparison between the linear and nonlinear processes with oscillatory, cosine-like inflow of $X$ reactant to the reaction medium. 
reason the analytical solution of this type equation is impossible. In the case of equation (4) it can only find a default solution of change of $X$ product. It is true, that the reaction (4) has two changes over time in oscillating manner stationary states:

$$
x_{1}=\frac{k_{1} A+\sqrt{\Delta}}{2 k_{2}} \text { and } x_{1}=\frac{k_{1} A-\sqrt{\Delta}}{2 k_{2}}
$$

where:

$$
\begin{aligned}
\sqrt{\Delta}= & \sqrt{\left(k_{1} A\right)^{2}+4 k_{2} a \cos ^{2}(\omega t)} \text { in case outfbw of } X, \\
& \Delta>0
\end{aligned}
$$

$$
\begin{aligned}
\sqrt{\Delta}= & \sqrt{\left(k_{1} A\right)^{2}-4 k_{2} a \cos ^{2}(\omega t)} \text { in case inflow of } X, \\
& \Delta>0 \text { or } \Delta<0
\end{aligned}
$$

The formula (5) contains changing time $t$ variable and it is not possible to solution. For this reason the eq. (4) is also impossible to analytical resolve. We assume that $\Delta$ is positive as in a constant inflow of $X$, because it has a definite physical meaning. Based on the analytical solution of nonlinear equation with a constant inflow of product $X$ to the reaction medium (see Appendix) and on the analytical solve of linear type equation with a periodically inflow of $X$ to the reaction medium described in our earlier paper and confirmed experimentally ${ }^{47}$ we assume that approximate solution of the eq. (4) can take the following form:

$$
\begin{aligned}
X= & \frac{k_{1} A-\sqrt{\Delta}-\left[\left(k_{1} A+\sqrt{\Delta}\right)\right] c \exp (\sqrt{\Delta} t)}{2 k_{2}[(1-c \exp (-\sqrt{\Delta} t)]} \\
& \times(\sqrt{\Delta} \cos 2 \omega t+2 \omega \sin 2 \omega t)
\end{aligned}
$$

Where, pre-exponential factor $c$ is,

$$
c=\frac{-X_{0} 2 k_{2}+k_{1} A-\sqrt{\Delta}}{-X_{0} 2 k_{2}+\left(k_{1} A+\sqrt{\Delta}\right) \sqrt{\Delta}}
$$

$X_{0}-$ is the initial concentration of the product $X . X_{0}$ is calculated from the following initial conditions: when $\mathrm{X}=\mathrm{X}_{0} \rightarrow t=0$ and it takes the form:

$$
X_{0}=\frac{k_{1} A-\sqrt{\Delta}-\left(k_{1} A+\sqrt{\Delta}\right) c \sqrt{\Delta}}{2 k_{2}-c 2 k_{2}}
$$

The relaxation time $\tau$ follows from the eq. (6) and takes the form:

$$
\tau=\frac{1}{\sqrt{\Delta}}=\frac{1}{\sqrt{\left(k_{1} A\right)^{2} \pm 4 k_{2} a \cos ^{2}(\omega t)}}
$$

As follows from eqs. (9) and (8) the determination of $\tau$ is impossible in this case, namely, nonlinear chemical processes with the oscillatory, cosine-like inflow to the reaction medium. The problem here is the time variable delta. This factor indicates the fundamental difference between linear and nonlinear reaction with oscillatory in time inflow of chemical reagent $X$ to the reaction medium.

As in the linear reaction with oscillatory inflow of $X$ to the reaction medium ${ }^{47}$ the sum of two trigonometric functions in brackets in eq. (6) can be replaced by a single trigonometric function after introducing an auxiliary angle $\phi$ defined as:

$$
\tan \phi=\frac{2 \omega}{\sqrt{\Delta}}=2 \omega \tau
$$

where,

$$
\phi=\arctan \frac{2 \omega}{\sqrt{\Delta}}=\arctan 2 \omega \tau
$$

After substitution eq. (11) into eq. (6) and some mathematical transformations we obtain:

$$
\begin{aligned}
X= & \frac{k_{1} A-\sqrt{\Delta}-\left[\left(k_{1} A+\sqrt{\Delta}\right)\right] c \exp (\sqrt{\Delta} t) \sqrt{\Delta}}{2 k_{2}[(1-c \exp (-\sqrt{\Delta} t)]} \\
& \times \frac{1}{\cos \phi} \cos (2 \omega t-\phi)
\end{aligned}
$$

where,

$$
\cos \phi=\frac{\sqrt{\Delta}}{\sqrt{(\sqrt{\Delta})^{2}+4 \omega^{2}}}
$$

Let us consider now a high-order autocatalysis level reaction type:

$$
A+2 X \stackrel{k_{1} k_{2}}{\longleftrightarrow} 3 X
$$

with a time constant inflow $I_{\mathrm{X}}$ of $X$ reagent to the reaction medium is described by the kinetic equation:

$$
\frac{d X}{d t}=k_{1} A X^{2}-k_{2} X^{3}+I_{X}
$$

The reaction has two stable stationary states. The analytical solution for these states is given by eq. (5).

When the inflow is oscillatory and time dependent, described by the eq. (3) approximate solution of the eq. (14) takes the form similar to eq. (6). The solution 
of eq. (14) applies only for one of the stable stationary state. It is known that the system tends to only one stable stationary state.

For the higher degrees of the nonlinear and oscillatory autocatalytic processes, seems to be that there is a dependency similar to eq. (2). For the higher degrees of autocatalysis in real space, i.e., three-dimensional space, there are only two stable steady states and the system seeks one of them.

It seems that the formula (6) also can apply to the one of the stable stationary state to the high-order autocatalytic reactions with the general formula:

$$
\frac{d X}{d t}=k_{1} A X^{(n-1)}-k_{2} X^{n}+a \cos ^{2}(\omega t)
$$

In general the formula (6) takes the form:

$$
\begin{aligned}
X= & \frac{k_{1} A-\sqrt[n]{\Delta}-\left[\left(k_{1} A+\sqrt[n]{\Delta}\right)\right] c \exp (\sqrt[n]{\Delta} t)}{2 k_{2}[(1-c \exp (-\sqrt[n]{\Delta} t)]} \\
& \times(\sqrt[n]{\Delta} \cos 2 \omega t+2 \omega \sin 2 \omega t)
\end{aligned}
$$

where, $n$ denotes the order of autocatalysis.

$X_{0}$ and $c$ are as described for $n=2$ by eq. (7) and eq. (8) but with $\sqrt[n]{\Delta}$.

The equation (4) can be numerically solved using four-order Runge-Kutta algorithm (figure 1). Figures $1 \mathrm{a}, 1 \mathrm{~b}$ and $1 \mathrm{c}$ present the numerical analysis (based on the Runge-Kutta algorithm) of a delay effect and an amplitude modification in the case of high-order nonlinear autocatalytic processes. All simulations were performed for the initial conditions: $X=1, t=0$ and an integration step $h=0.01$. The numerical calculations show a delay between the concentration of inflow product $X$ to the reaction medium (field) and its concentration in this medium. The amplitude of $X$ in the reaction field is modified too. The phase shift and amplitude modification depends on frequency $\omega$ of $X$ inflow, as well as on values of $k_{1} A$ and $k_{2}$. For a given level of autocatalysis the numerical solution shows the following dependence: when the influx of reagent $X$ to the field (medium) reaction is more intense, the delay in concentration in the reaction field is less or unchanged. (figures 1a-c, 2a-b, 3a-b and table 2).

In the linear type reaction with oscillatory, cosinelike inflow of the intermediate product $X$ to the reaction medium (field), the analytical solution of linear kinetic equation shows that the delay is ruled by the formula: $\tan \Phi=\omega t .{ }^{47}$ This formula was confirmed experimentally and was used to determination of the rate constant $k$ of the saponification of ethyl acetate (used as a reagent $A$ ) with sodium hydroxide (used as a reagent $X$ ).
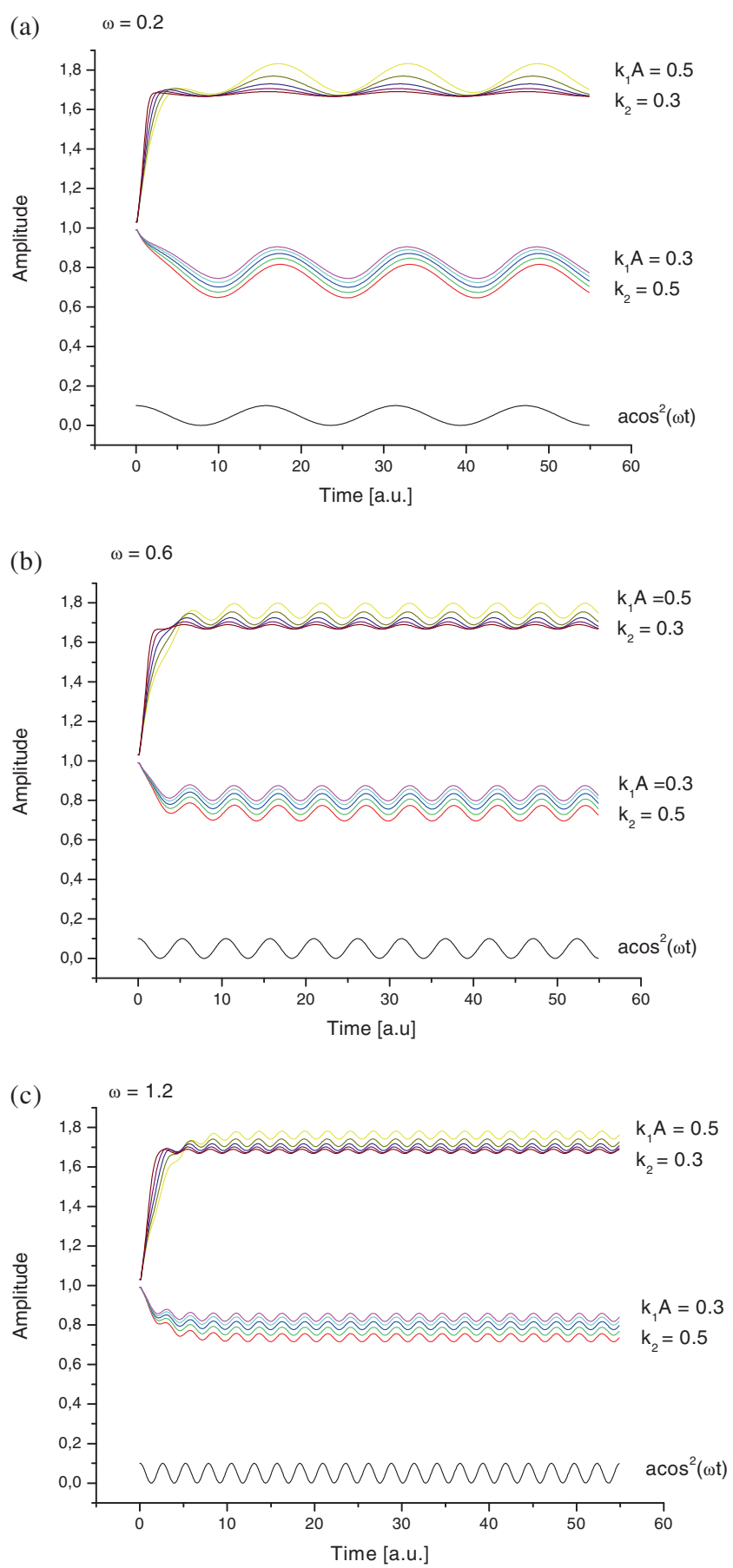

Figure 1. The results of numerical calculation for the autocatalytic reactions with oscillatory stimulus (described by eq. (4)) both for $k_{1} A>k_{2}$ (upper lines) and $k_{1} A<k_{2}$ (lower lines) for a) $\omega=0.2$, b) 0.6 and c) 1.2 . For $k_{1} A<k_{2}$ (lower lines) from the bottom, the lines correspond $2 \mathrm{X}, 3 \mathrm{X}, 4 \mathrm{X}, 5 \mathrm{X}$ and $6 \mathrm{X}$; for $k_{1} A>k_{2}$ (upper lines) from the top (upper lines), the lines correspond $2 \mathrm{X}, 3 \mathrm{X}, 4 \mathrm{X}, 5 \mathrm{X}$ and $6 \mathrm{X}$. The black line at the bottom of figure is a periodical, cosine-like inflow of the intermediate product $X$. The calculation parameters are initial concentration of $X: X_{0}=1$, initial time: $t_{0}=0$, integration step $h=0.01, k_{1} A=0.5, k_{2}=0.3, a=0.1$. 

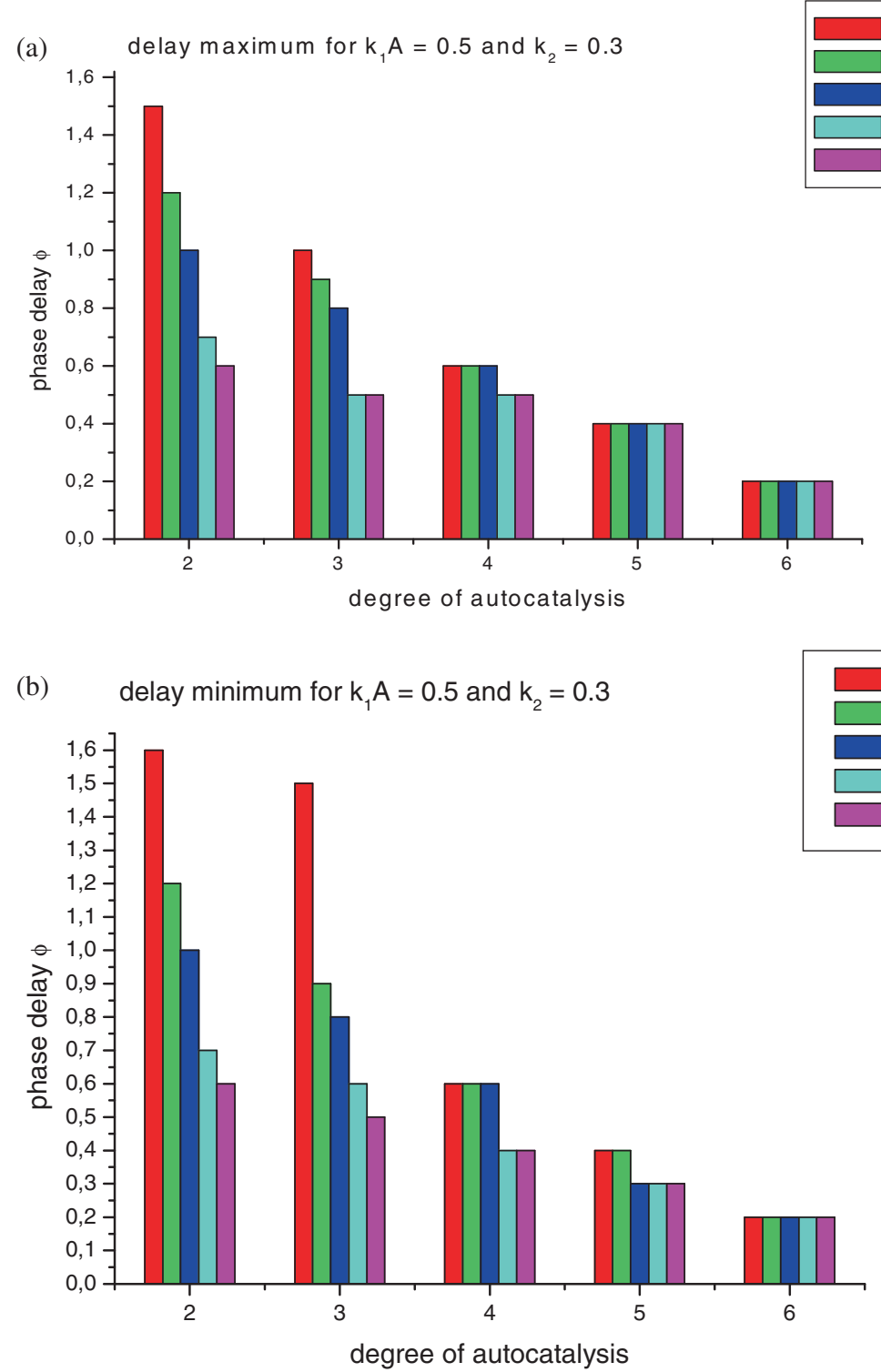

Figure 2. Illustration of a) the phase delay $\phi$ maximum and b) the phase delay minimum, dependence on frequency $\omega ; 0.2,0.4,0.6,0.9,1.2$ of the $X$ inflow and on autocatalysis degree for $k_{1} A>k_{2}: k_{1} A=0.5, k_{2}=0.3$.

In the presented model $\sqrt{\Delta}$ probably depends on the order of autocatalysis in case when $\sqrt{\Delta}$ is positive this is, for $k_{1} A>k_{2}$. For higher levels of autocatalysis a sign of the delta (positive or negative) probably determines whether a system oscillations disappear or not (at a fixed frequency inflow of $X$ reagent, see figures $1 \mathrm{a}-\mathrm{c}$,). When the $k_{1} A$ is greater than $k_{2}$ (delta is positive) the damping of the amplitude of the oscillations and decrease in the phase shift are observed in the system with an increase in the degree of autocatalysis (figures 1a-c (upper lines) and figures $2 \mathrm{a}-\mathrm{b}$ and table 2) (the shift between the oscillatory inflow of $X$ reagent to the reaction medium and its concentration in this medium). This is visible both in maximum and minimum of $X$ inflow (figures $2 \mathrm{a}-\mathrm{b}$ ). As shown in the figures $2 \mathrm{a}$ and $2 \mathrm{~b}$, when the $k_{1} A$ is greater than $k_{2}$ the phase shift depends strictly on the frequency $\omega$ of supply of $X$ reagent to the reaction medium - decreases with increasing of $\omega$ inflow of reactant $X$ at the given degree of autocatalysis. As shown in the figures $2 \mathrm{a}$ and $2 b$, with the increase in the degree of autocatalysis, phase shift between the concentration of the reactant $X$ and the inflow is reduced. At six-order of autocatalysis the phase shift does not seem to depend on frequency $\omega$ of flow of $X$. A similar correlation is observed in the amplitude values obtained from numerical calculation as show in table 2 . When the $k_{1} A$ is smaller than the $k_{2}$ ( $\Delta$ is negative) the oscillation of $X$ are sustained (with 

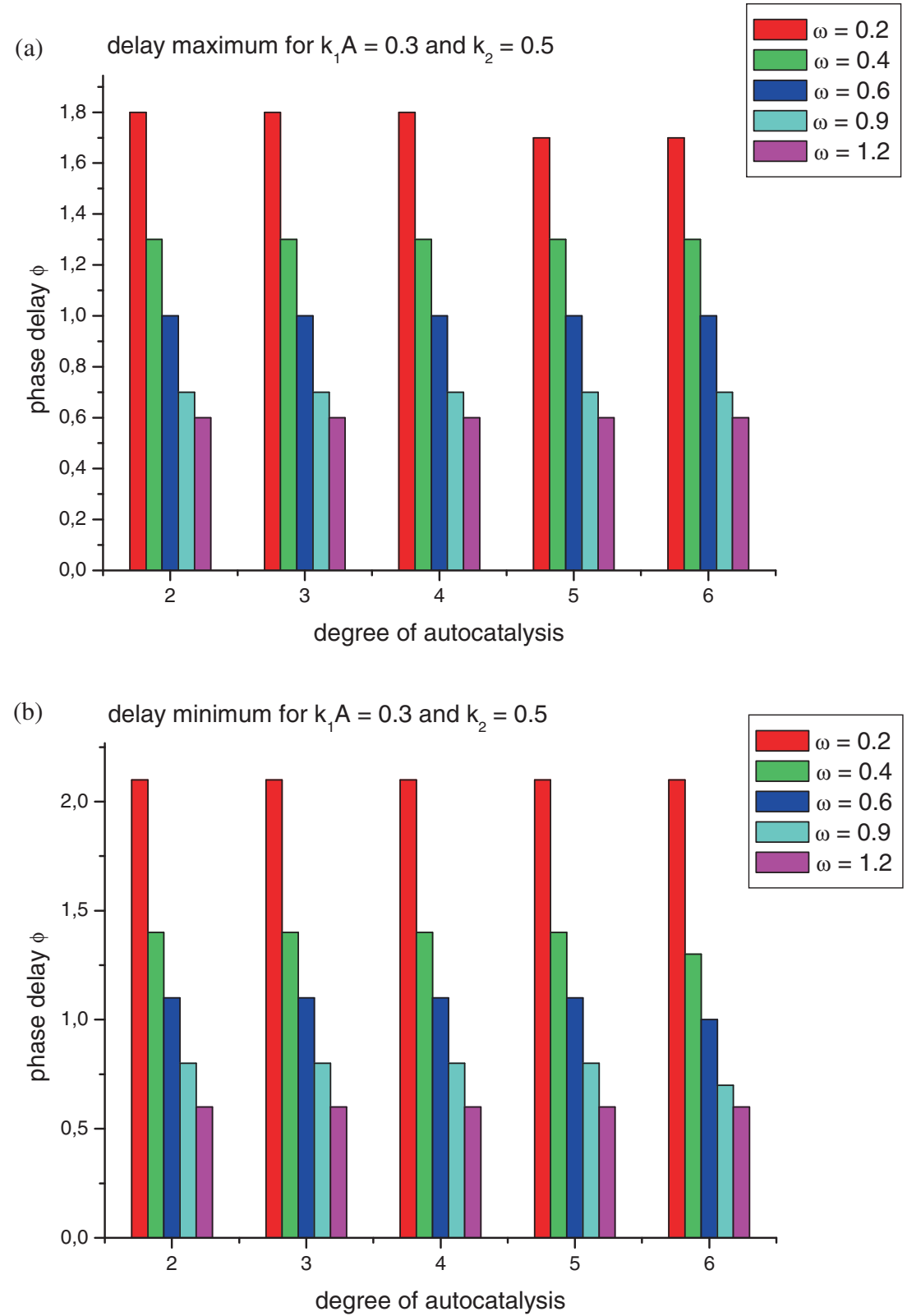

Figure 3. Illustration of a) the phase delay $\phi$ maximum and b) the phase delay minimum, dependence on frequency $\omega ; 0.2,0.4,0.6,0.9,1.2$ of the $X$ inflow and on autocatalysis degree for $k_{1} A<k_{2}: k_{1} A=0.3, k_{2}=0.5$.

Table 2. Juxtaposition of the values of amplitude obtained from the numerical calculations for the case: $k_{1} A>k_{2}, k_{1} A=0.5, k_{2}=0.3$. $\omega$ is a frequency of $X$ supply, $2 \mathrm{X}, 3 \mathrm{X}, 4 \mathrm{X}, 5 \mathrm{X}$ and $6 \mathrm{X}$ denote the degree of autocatalysis.

\begin{tabular}{lccccc}
\hline Degree of autocatalysis & $\omega=0.2$ & $\omega=0.4$ & $\omega=0.6$ & $\omega=0.9$ & $\omega=1.2$ \\
\hline $\mathbf{2 X}$ & 0.14 & 0.1 & 0.08 & 0.05 & 0.04 \\
$\mathbf{3 X}$ & 0.1 & 0.08 & 0.07 & 0.04 & 0.03 \\
$\mathbf{4 X}$ & 0.06 & 0.06 & 0.05 & 0.04 & 0.03 \\
$\mathbf{5 X}$ & 0.03 & 0.03 & 0.03 & 0.02 & 0.02 \\
$\mathbf{6 X}$ & 0.02 & 0.02 & 0.02 & 0.02 & 0.01 \\
\hline
\end{tabular}

increase of degree of autocatalysis ((figure 1a), figure 1b), figure 1c) - lower graphs)) and the phase delay does not change with increasing of degree of autocatalysis at the given frequency $\omega$ of $X$ inflow (figures $3 \mathrm{a}$ 
and $3 \mathrm{~b})$. But, in this case, the phase shift depends on frequency $\omega$ of $X$ inflow at the given degree of autocatalysis (figures $3 \mathrm{a}$ and $3 \mathrm{~b}$ ). In both cases (when $k_{1} A$ is greater than $k_{2}$ or $k_{1} A$ is smaller than the $k_{2}$ ) the phase shift depends on frequency $\omega$ of inflow of $X$ at the given degree of autocatalysis. When the frequency $\omega$ of $X$ inflow to the reaction medium is greater, the phase shift (phase delay) of the concentration of $X$ in this medium is reduced at a given order of autocatalysis as shown in the figure 2 and figure 3 . It is opposite to the effect in linear reactions. ${ }^{47}$ Apart from that, bifurcation point is visible in figures $1 \mathrm{a}-\mathrm{c}$.

\section{Results and Discussion}

\subsection{Physical interpretation - connection between the exponential relaxation and the forced delay effect in the linear and nonlinear systems and some examples}

In nature the most of relaxation processes have an exponential character. In the biological and chemical systems, relaxation takes place exponentially in almost 70-80\%. In chemistry the first order and autocatalytic non oscillating type of reactions have an exponential relaxation. Especially the nonlinear autocatalytic processes play an important role in biological systems.

If (in the non-oscillating and exponentially relaxing systems) intermediate product $X$ flows in the oscillatory manner to the reaction medium (as a variable time cosine type function) it will be seen the phase shift in the concentration of $X$ in response to the supply of this component to the medium. This process is called as a forced (by the oscillating stimulus), the effect of phase shift. Phase shift (phase delay) is strictly connected with the relaxation process.

There is a one common formula for all oscillating and non-oscillating linear systems relaxing exponential and forced by a periodical stimulus: $\tan \Phi=\omega t$, where $\omega-$ is a frequency of stimulus, $\tau-$ is a relaxation time. This formula is mainly used for determination of the relaxation time $\tau$, especially in physical systems. In the linear first-order chemical reactions, the forced phase delay effect turned out to as a good method for determination of the rate constant $k_{1}$ and the relaxation time $\tau{ }^{47}$

There are common factors relating to all the linear relaxing exponentially systems both oscillatory and non oscillatory forced by oscillatory stimulus. Parameters of the forced oscillation do not depend on the initial conditions. These oscillations have the frequency of driving force and are characterized by amplitude and phase shift (with respect to the appropriate stimulus).

There are some differences characterizing the nonlinear systems with respect to the linear ones. There are the common characteristic for the nonlinear systems, both oscillatory and non oscillatory, forced by oscillatory stimulus. In this work we present the non-oscillating and nonlinear system forced by oscillatory stimulus. Parameters of the forced oscillation strictly depend on the initial conditions. Nonlinear systems are sensitive to the initial conditions. The oscillations have the frequency of driving force and are characterized by amplitude and phase shift (as in the linear systems). There is no one set rule which describes the behaviour of the nonlinear systems. This is due to the fact that the nonlinear systems are far from thermodynamic equilibrium.

The forced phase shift in the nonlinear systems is even less frequent than in the linear ones. There are known examples of nonlinear physical systems in which there is a forced phase shift effect. It is difficult to find examples of nonlinear chemical systems in which the forced phase shift effect is observed. A special class of reaction-diffusion equations to a system incorporating time delay effects were theoretically considered. ${ }^{48}$ The equations applied e.g., to a nonlinear reaction-diffusion Lotka-Volterra system.

\subsection{Discussion}

The nonlinear, non-oscillating and an auto-processingautocatalytic chemical reactions are considered in this paper. The simplest nonlinear autocatalytic type reaction with the oscillatory inflow of $X$ reagent to the reaction medium (reaction field) is much more complicated in comparison to the linear first-order reaction with the $X$ reagent inflow to the reaction medium. In our case, the delay in the concentration of $X$ chemical reagent in the reaction medium is connected with its frequency of inflow to the reaction medium. As in the linear first-order process we also observed the phase shift between the $X$ inflows to the reaction medium and change its concentration in this medium. The amplitude modification is observed too. However in the nonlinear autocatalytic processes the $\Delta$ is connected with an oscillatory time dependent inflow of $X$ and is variable in time (Eqs. 5a and 5b). Connected with the $\Delta$ the relaxation time $\tau$ is also variable in time. Apart from $\Delta$ and $\tau$ are also dependent on $k_{1} A$ and $k_{2}$. For this reason the relaxation time $\tau$ as well as $k_{1} A$ and $k_{2}$ are not possible to determination. Nonlinear autocatalytic processes of high-order have also a one stable stationary state, which rule this same or similar formula as the simplest autocatalytic process one. Determination of the relaxation time $\tau$ and rate constants $k_{1}$ and $k_{2}$ are impossible too.

We observe that with increasing frequency of $X$ inflow to the reaction medium the phase shift tends to 
zero. In the linear first-order reaction, the phase shift tends to $\pi / 2$ with increasing frequency of $X$ inflow. It may be connected with the fundamental difference between the systems linear and nonlinear. It is probably connected with $\Delta$ oscillating in time. The fact that the phase shift decreases with increasing $\omega$ inflow $X$ seems to be important especially in the biological systems. High frequency $\omega X$ component supply needed for the occurrence of a chemical reaction reduces the delay of its delivery. This is very important for the proper conduct of the chemical process in a living organism. Most nonlinear biological processes, such as, enzymatic reactions, DNA replication, biosynthesis of protein and many others occurs with very great speed of $10^{12} \mathrm{~s}$. It follows that the reagent $X$ must also be provided at the same rate. The rate is 1000 times greater than the rate of transmission of any signal, which is $10^{9} \mathrm{~s}$. When the delivery rate of reagent $X$ is very rapid, about $10^{12}$ $\mathrm{s}$, a delay in phase is observed. Also, any oscillations are extinguished. In the linear reactions it is inverse; the higher the frequency of reactant $X$ supply to the reaction medium, the greater is the phase delay, and tends to a maximum of $90^{\circ}$ (but does not exceed this value). This explains the very important role of nonlinearity in biology. For a biological system to function effectively there can be no delay in the chemical process. If the reactant flow rate decreases, the delay may occur, which may have an adverse effect on the functioning of the biological system. Presented model explains the behavior of the nonlinear system, depending on the frequency of $X$ reagent flow to the reaction field. In some cases, when $k_{1} A<k_{2}$ ( $\Delta$ probably is negative) the oscillations are sustained in system. This fact is also important in the living systems that allows to preserve stability.

Table 1 presents a comparison between the linear and nonlinear chemical processes referring to the forced phase delay effect. Large differences are apparent between the linear first order chemical reactions with the oscillatory inflow of $X$ reactant and the nonlinear autocatalytic ones with the same inflow. It is a fundamental difference between the linear chemical reactions with forced delays effect ${ }^{47}$ (table 1). In the linear first-order reactions with an oscillatory inflow of $X$ reagent to the reaction medium there is only one stationary state and there is only one analytical solution. The relaxation time $\tau$ is strictly connected with $k_{1} A$ and $\tan \phi=2 \omega \tau$. In the nonlinear processes with the oscillatory inflow of $X$ reagent to the reaction medium is much more complicated. In the first-order linear reactions with oscillatory inflow of $X$ to the reaction medium, the phase delay $\phi$ is greater with increase of frequency $\omega$ of its inflow. In the autocatalytic nonlinear reactions, the phase shift is reduced with an increase in frequency $\omega$ of $X$ inflow to the reaction medium (figures $2 \mathrm{a}$ and $2 \mathrm{~b}$, figures $3 \mathrm{a}$ and $3 \mathrm{~b}$ ) at a given degree of autocatalysis. For the higher order of autocatalysis (five and six), the phase delay seems to not depend on frequency $\omega$ of $X$ inflow in some cases (when $k_{1} A>k_{2}$, see figures $2 a$ and $2 b$ ).

The following question arises: is it possible that any application of the forced phase delay affect the nonlinear autocatalytic processes? In order to answer this question let's cite one researcher - Laister. Laister ${ }^{48}$ in the abstract of his article writes:

"For a significant class of nonlinear equations it is shown that an unstable equilibrium solution of the reaction-diffusion system cannot be stabilized by the introduction of delay". ${ }^{48}$ In our case the nonlinear autocatalytic but without diffusion processes, cannot be stabilized by forced delay effect. For this reason the forced delay effect in the nonlinear chemical processes cannot be applied for determination of a rate constant or a relaxation time.

The phase delays' phenomena seem also to play an important role in some biological processes. It has been proved that the $\mathrm{p} 19$ protein induces G1/S phase delay, thereby maintaining cells in a reversible quiescence state and preventing entry into apoptosis ${ }^{49} \mathrm{~N}$-methyl-D Revised: aspartate (NMDA) induces a rapid phase shift of mPer 1promotor gene in cultured SCN. It has been shown that NMDA induces phase shifts at various time points. ${ }^{50}$

Autocatalytic processes are connected with the class of reactions which are most closely associated with creation. Stuart Kauffman in his book wrote that life initially arose as autocatalytic chemical networks. ${ }^{51}$

The theoretical model presented in this paper can also be applied to the theory of heterogeneous catalysis. In practice, the present model does not apply here, because of the intermediate species which are not available outside reactor. But this model may be useful to explain the role of autocatalytic stage occurring during heterogeneous catalysis.

\section{Conclusions}

Our theory shows that in the nonlinear and nonoscillating chemical processes, the phase shift and oscillation amplitude decrease with increasing flow frequency of the intermediate product $X$ to the reaction medium. For this reason, the forced phase delay effect seems to play an important role in all nonlinear processes in biological systems.

The phase delay effect (phase shift) in the nonlinear and non-oscillating chemical processes forced by 
the oscillatory inflow of chemical reagent cannot be used for determination of both a rate constant and a relaxation time because of a time variable $\Delta$.

It seems that there is one common mathematical formula possible to describe nonlinear autocatalytic processes of all orders.

\section{Acknowledgements}

I would like to thank Prof. Jerzy Konarski of Adam Mickiewicz University in Poznan for the initiation of the topic of the mathematical model of oscillatory flow of reagent $\mathrm{X}$ to the reaction field in the nonlinear chemical reactions, for writing a program to solve nonlinear differential equations based on Runge-Kutta algorithm and some useful discussions.

\section{Appendix}

Derivation of the approximate solution of the irresolvable nonlinear equation (eqs. (6) and (16)) in this work are based on analytical calculation for the nonlinear autocatalytic reaction with a constant in time inflow of the reactant $X$ to the reaction medium and on the analytical solution for the simple linear reaction with oscillatory, cosine-like inflow of $X$ to the reaction medium described in the ref. ${ }^{47}$

Derivation of Eq. (6) and (16):

The main subject of our consideration is the simplest nonlinear autocatalytic reaction:

$$
A+X \stackrel{k_{1} k_{2}}{\longleftrightarrow} 2 X \quad I_{X}=\text { const. }
$$

where $A$ stands for the initial product, $X$ is the intermediate product, $I_{\mathrm{X}}$ denotes constant in time inflow of the intermediate product $X$ to the reaction field (medium).

We assume that the product $X$ flow to the reaction medium If the inflow $I_{X}$ of intermediate product $X$ is constant in time, the kinetic equation (1) has the form:

$$
\frac{d X}{d t}=k_{1} A X-k_{2} X^{2}+I_{X}
$$

The reaction (2) has the following stationary states:

$$
x_{1}=\frac{k_{1} A+\sqrt{\Delta}}{2 k_{2}} \quad x_{1}=\frac{k_{1} A-\sqrt{\Delta}}{2 k_{2}}
$$

where:

$$
\begin{gathered}
\sqrt{\Delta}=\sqrt{\left(k_{1} A\right)^{2}+4 k_{2} I_{X}} \text { in case outflow of } X \Delta>0 \\
\text { is always positive } \\
\sqrt{\Delta}=\sqrt{\left(k_{1} A\right)^{2}-4 k_{2} I_{X}} \text { in case inflow of } \\
X \Delta>0 \text { or } \Delta<0
\end{gathered}
$$

We assume that $\Delta$ is positive.

The analytical solution of the $X$ product change described by equation (2) takes the form:

$$
X=\frac{k_{1} A-\sqrt{\Delta}-\left[\left(k_{1} A+\sqrt{\Delta}\right)\right] c \exp (\sqrt{\Delta} t)}{2 k_{2}[(1-c \exp (\sqrt{\Delta} t)]}
$$

where, pre-exponential factor $c$ is,

$$
c=\frac{-X_{0} 2 k_{2}+k_{1} A-\sqrt{\Delta}}{-X_{0} 2 k_{2}+k_{1} A+\sqrt{\Delta}}
$$

$X_{0}$ - is the initial concentration of the product $X . X_{0}$ is calculated from the following initial conditions:

when $\mathrm{X}=\mathrm{X}_{0} \rightarrow t=0$ and $X_{0}$ takes the form:

$$
X_{0}=\frac{k_{1} A-\sqrt{\Delta}-\left(k_{1} A+\sqrt{\Delta}\right) c}{2 k_{2}-c 2 k_{2}}
$$

From the equation (4) follows the relaxation time $\tau$ for this reaction which takes the form:

$$
\tau=\frac{1}{\sqrt{\Delta}}=\frac{1}{\sqrt{\left(k_{1} A\right)^{2}-4 k_{2} I_{X}}}
$$

Analytical solution for the linear reactions with oscillatory, cosine-like inflow of intermediate product $X$ to the reaction medium is described in our earlier paper (see ref. $\left.{ }^{47}\right)$.

\section{References}

1. Nicolis G, Prigogine I 1977 In Self-organization in non-equilibrium systems. From dissipative structures to order through fluctuations (New York: Wiley and Sons)

2. Scott S K 1991 In Chemical chaos (Oxford: Clarendon Press)

3. Paul N and Joyce G F 2002 Proc. Natl. Acad. Sci. USA 912733

4. Lee D H, Granja J R, Martinez J A, Severin K and Ghadri M R 1996 Nature 382525

5. Eigen M 1996 Biophys. Chem. 63 A1-18

6. Witzemann E J 1924 J. Phys. Chem. 28305

7. Drummond P D, Vaughan T G and Drummond A 2010 J. Phys. Chem. 11410481

8. Pompano R R, Li H-W and Ismagilov R F 2008 Biophys. J. 91531

9. Toth R, Gaspar V, Belmonte A, O'Connell M C, Taylorc A and Scott S K 2000 Phys. Chem. Chem. Phys. 2413

10. Wu Y, Vasquez D A, Edwards B F and Wilder J W 1995 Phys. Rev. E 511119

11. Belousov B P 1985 In A Periodic Reaction and Its Mechanism in Oscillations and Traveling Waves in Chemical Systems. R J Field, M Burger (eds.) (New York: Wiley) pp. 605-613

12. Benini O, Cervellati R and Fetto P 1996 J. Chem. Ed. 73 865 
13. Vanag V K, Zhabotinsky A M and Epstein I R $2000 \mathrm{~J}$. Phys. Chem. A 10411566

14. Asai T, Kanazawa Y, Hirose T and Amemiya Y 2005 Int. J. Unconv. Comput. 1123

15. Qiao Ch, Wang H and Ouyang Qi 2009 Phys. Rev. E 79 016212

16. Flesselles J M, Belmonte A and Gaspar V 1998 J. Chem. Soc. Faraday Trans. 94(7) 851

17. Chance B, Pye E K Ghosh A K and Hess B 1973 In Biological and Biochemical Oscillators (New York: Academic Press)

18. Lengyel I, Nagy I and Bazsa G 1989 J. Phys. Chem. 93 2801

19. Edblom E C, Orbán M and Epstein I R 1986 J. Am. Chem. Soc. 1082826

20. Showalter K 1981 J. Phys. Chem. 85(4) 440

21. Gribschaw T A, Showalter K, Banvilla D L and Epstein I R 1038 J. Phys. Chem. 82152

22. Gowland R J and Stedman G J 1983 Chem. Soc. Chem. Commun. 1038

23. Bazsat G and Epstein I R 1985 J. Phys. Chem. 83050

24. Sakai K, Rien S and Schwartz L B 1996 J. Clin. Invest. 97988

25. Dateo C E, Orbán M, De Kepper P and Epstein I R 1982 J. Am. Chem. Soc. 104504

26. Paul N and Joyce G F 2004 Chemical Biology 8634

27. Field R J and Noyes R M 1974 J. Am. Chem. Soc. 96 2001

28. Showalter K, Noyes R M and Turner H 1979 J. Am. Chem. Soc. 1017463

29. Jorne J 1980 J. Am. Chem. Soc. 1026196

30. Gribschaw T A, Showalter K, Banville D L and Epstein I R 1981 J. Phys. Chem. 852152

31. Weitz D M and Epstein I R 1984 J. Phys. Chem. 885300

32. Szirovicza L, Nagypal I and Boga E $1989 \mathrm{~J}$. Am. Chem. Soc. 1112842

33. Reusser E J and Field R J 1979 J. Am. Chem. Soc. 101 1063
34. Decroly O and Goldbeter A 1985 J. Theoret. Biol. 113 649

35. Decroly O and Goldbeter A 1987 J. Theoret. Biol. 124 219

36. Decroly O and Goldbeter A 1984 Physics Letters 105A 259

37. Györgi L and Field R J 1992 Nature 355808

38. Eigen M and Schuster P 1979 In The Hypercycle: A Principle of Natural Self-Organization (Berlin: Springer-Verlag)

39. Petrov V, Scott S K and Showalter K 1992 J. Chem. Phys. 976191

40. Rlnzel J and Ermentrout G B 1982 J. Phys. Chem. 86 295441

41. Hanna A, Saul A and Showalter K 1982 J. Am. Chem. Soc. 1043838

42. Gáspár V and Showalter K 1990 J. Phys. Chem. 944973

43. Aduda B D and Larter R 1989 J. Chem. Phys. 904168

44. Ortega L A 1998 Journal of Mathematical Analysis and Applications 221712

45. Sanchirico R 2012 AIChe Journal 581869

46. Sumarni A B and Razidah I 2014 In Proceedings of the 2014 International Conference on Mathematical Methods, Mathematical Models and Simulation in Science and Engineering 123 ISBN: 978-1-61804-219-4

47. Krupska A, Konarski J, Fiedorow R and Adamiec J 2002 Kinetics and Catalysis $\mathbf{4 3} 295$

48. Laister R 2000 Journal of Mathematical Analysis and Applications 247588

49. Camats M, Kokolo M, Heesom K J, Ladomery M and Bach-Elias M 2009 Stat PLoS ONE 41

50. Asai M, Yamaguchi S, Isejima H, Jonouchi M, Moriya T, Shibata S, Kobayashi M and Okamura H 2001 Current Biology 111524

51. Kauffman S 1995 In At Home in the Universe: The Search for the Laws of Self-Organization and Complexity (Oxford: University Press) (ISBN 0-19509599-5) 